\title{
HERNIA DE LITTRE EN LACTANTE MENOR
} LITTRÉ HERNIA AS AN INCIDENTAL FINDING IN A YOUNG INFANT HÉRNIA DE LITTRE COMO UM ACHADO INCIDENTAL EM UM BEBEE PEQUENO

\author{
JOHNSON SUÁREZ VELOZ', CRISTHIAN ASPIAZU², DIANA GAMBOA PARRA³, STEFANIA OYARVIDE PALOMINO³, JHONNY \\ HIDROVO 4 \\ Ministerio de Salud Pública de Ecuador. \\ Hospital León Becerra, Guayaquil, Ecuador \\ Universidad de Guayaquil; Guayaquil, Ecuador \\ 4 Hospital del Instituto Ecuatoriano de Seguridad Social, Guayaquil, Ecuador
}

\begin{abstract}
La hernia de Littré es la presencia del divertículo de Meckel en cualquier saco herniario. Su incidencia es desconocida y se han descrito 69 casos en la literatura inglesa hasta el 2011. Se presenta el caso de un lactante menor masculino de 3 meses de edad con la presencia de una masa inguinal derecha reductible. La cirugía programada fue herniectomía inguinal de forma ambulatoria. Durante el transoperatorio se encontró un divertículo de Meckel dentro del saco herniario inguinoescrotal, se realizó herniorrafía inguinal derecha. El paciente es dado de alta a las 2 horas de postoperatorio sin complicaciones.
\end{abstract}

PALABRAS CLaVE: hernia de Littré, divertículo de Meckel, lactante menor.

ABSTRACT

Littré hernia is the presence of a Meckel diverticulum in any hernial sac. Its incidence is unknown and, 69 cases have been described in the English literature until 2011. We present a case report of a 3-month-old male infant with the presence of a reducible right inguinal mass. The scheduled surgery was inguinal herniotomy on an outpatient basis. During the intraoperative period, Meckel diverticulum was found within the inguinoscrotal hernial sac, a right inguinal herniorrhaphy was performed. The patient is discharged 2 hours after surgery without complications. Keywords: Littré hernia, Meckel diverticulum, young infant. Littré hernia as an incidental finding in a young infant.

KEYWORDS: littré hernia, Meckel diverticulum, infant..

RESUMO

A hérnia de Littré é a presença do divertículo de Meckel em qualquer saco herniário. Sua incidência é desconhecida e 69 casos foram descritos na literatura inglesa até 2011. Apresentamos 0 caso de um lactente de 3 meses de idade com presença de massa inguinal direita redutível. A cirurgia programada foi herniectomia inguinal em regime ambulatorial. No intra-operatório, foi encontrado divertículo de Meckel no interior do saco herniário inguinoescrotal, foi realizada herniorrafia inguinal direita. 0 paciente recebe alta 2 horas após a cirurgia sem complicações. Palavras-chave: hérnia de Littré, divertículo de Meckel, lactente.

PALAVRAS-CHAVE: littré hérnia, divertículo ileal, lactante. 


\section{INTRODUCCIÓN}

La hernia de Littré consiste en la presencia del divertículo de Meckel en cualquier saco herniario. ${ }^{1}$ Esta entidad clínica fue descrita en 1700 por Alexis de Littre luego de los hallazgos de autopsia en dos pacientes y las describió originalmente como la presencia de un divertículo ileal en una hernia inguinal. El término hernia de Littre fue aceptado casi un siglo después luego de que Frederich Meckel definiera un verdadero divertículo congénito del intestino del cual lleva su nombre. No se conoce realmente su incidencia a nivel mundial. En la literatura inglesa hasta el 2011 se han reportado 69 casos de hernia de Littré en niños. ${ }^{2}$

Presentamos el caso de un lactante menor masculino con hernia de Littré como hallazgo intraoperatorio y se realiza una revisión bibliográfica.

\section{CASO CLÍNICO}

Lactante menor masculino de 3 meses de edad que cursa cuadro clínico de 2 meses de evolución caracterizado por la presencia de una protrusión reductible a nivel inguinal derecha que se extiende hasta el escroto la cual aumenta con el llanto. Como antecedentes familiares y prenatales: padres aparentemente sanos, abuelos con diabetes mellitus tipo II, no refieren enfermedades congénitas en el grupo familiar. Los antecedentes obstétricos: madre de 19 años de edad, primigesta, primípara, embarazo controlado mensualmente en otra institución hospitalaria, anemia a las 18 semanas de gestación, infecciones del tracto urinario en el último trimestre del embarazo. Antecedentes natales: producto obtenido por parto eutócico a las 39 semanas de gestación, APGAR 9/10, peso 3,500g, talla $55 \mathrm{~cm}$, perímetro cefálico $35 \mathrm{~cm}$.

El examen físico: peso $5.2 \mathrm{~kg}$, talla $60 \mathrm{~cm}$, percentil 10-25 para la edad, eutrófico, normorreactivo. Se palpa masa inguinal derecha reductible que se extiende hasta el escroto que aumenta con maniobras de Valsalva. La biometría hemática completa y los tiempos de coagulación fueron normales por lo que la cirugía programada fue herniectomía inguinal de forma ambulatoria con diagnóstico preoperatorio de hernia inguinoescrotal derecha. Durante el transoperatorio se encontró un divertículo de Meckel, a $60 \mathrm{~cm}$ de la válvula ileocecal de aspecto normal sin complicaciones, dentro del saco herniario inguinoescrotal derecho, se realizó herniorrafía inguinal derecha sin la resección del divertículo de Meckel. El pa- ciente es dado de alta a las 2 horas de postoperatorio sin complicaciones. Se prescribió paracetamol vía oral para analgesia postoperatoria y controles subsecuentes cada 2 meses. Al momento cursa un año de postoperatorio asintomático.

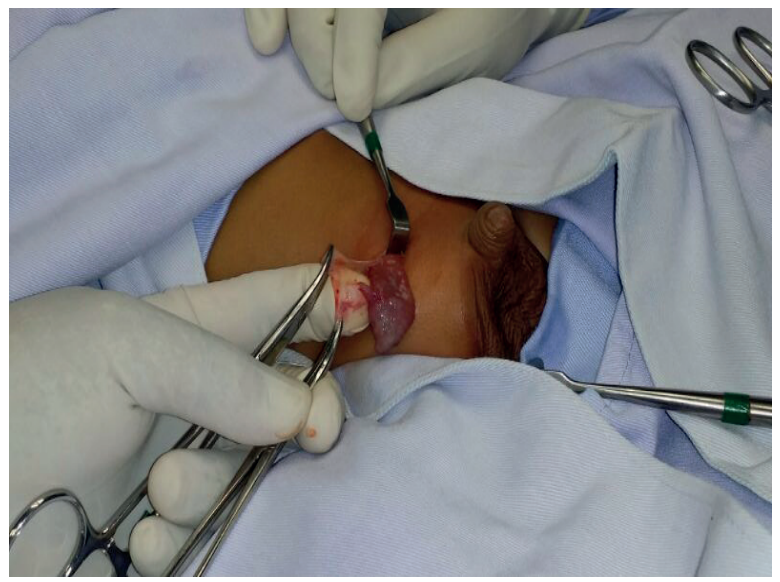

Figura 1. Hernia de Littré en lactante menor (Suarez, J; 2014).

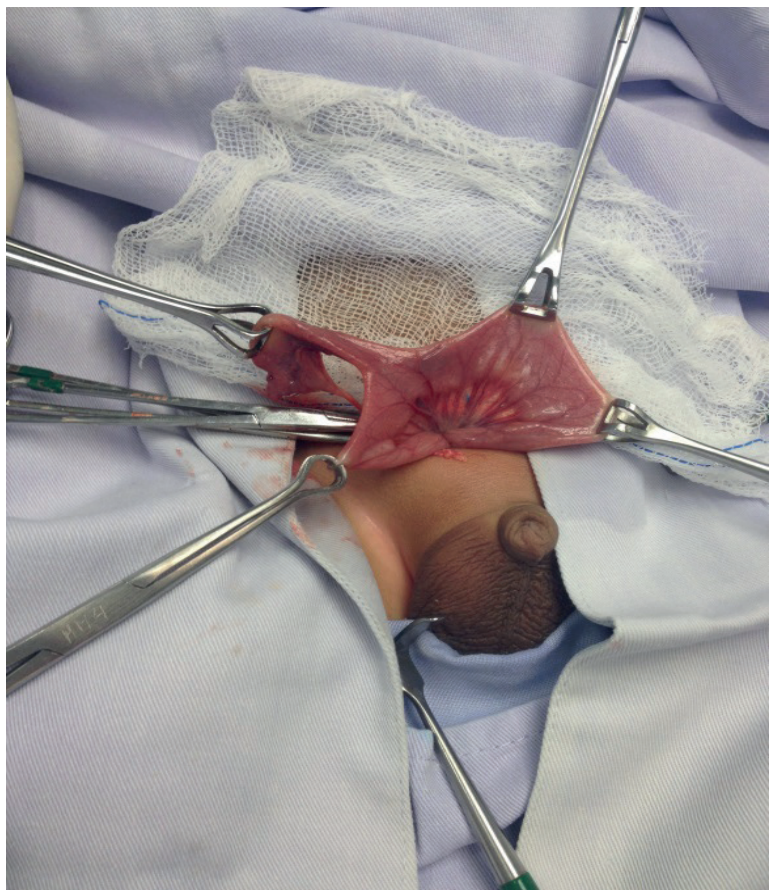

Figura 2 Divertículo de Meckel en una hernia inguinal. Hernia de Littré (Suarez, J; 2014)

DISCUSIÓN

En 1745 el anatomista y cirujano francés Alexis Littré describió la presencia del divertículo de Meckel en una hernia. ${ }^{3}$ La incidencia de esta patología aún es desconocida y la mayoría de las publicaciones son reportes de casos. Se han descrito varias localizaciones de la hernia de Littré, las más habituales son: inguinal $50 \%$, Umbilical 20\% y Femoral 20\%.4,5 También se han reportado casos en hernia diafragmática ${ }^{2}$, hernia incisional ${ }^{6}$. Ahmad y cols. reportaron en el 2006 
un raro caso de hernia de Littre en una hernia incisional por puerto laparoscópico. ${ }^{7}$ En la edad adulta la localización más frecuente de la Hernia de Littré es en la región inguinal, mientras que en los niños es a nivel umbilical reportándose una incidencia del $85 \% .{ }^{2}$ Por su baja incidencia el diagnóstico preoperatorio es complejo, generalmente insospechado. Los síntomas son inespecíficos y generalmente su diagnóstico se lo realiza durante la cirugía.

El tratamiento es quirúrgico y comprende la resección del divertículo y reparo de la hernia. ${ }^{8}$ El manejo quirúrgico del divertículo de Meckel asintomático hallado en forma incidental sigue siendo controversial. ${ }^{9}$ La morbimortalidad de la diverticulectomía del Meckel incidental debe compararse con la probabilidad del que divertículo produzca síntomas. ${ }^{10}$ Se estima que el riesgo durante toda la vida de que un divertículo de $\mathrm{Mec}^{-}$ kel se complique es del 4 al $6 \%$, por lo que no todos los divertículos asintomáticos deben extirparse ${ }^{10}$ a diferencia de si se presentan complicaciones del divertículo de Meckel, el tratamiento es la resección quirúrgica. ${ }^{9}$

Las opciones quirúrgicas para la resección del divertículo de Meckel incluyen la diverticulectomía o la resección del segmento intestinal y anastomosis primaria. ${ }^{11}$ De acuerdo a Dunn y Markgraf, la resección del divertículo se justifica por el riesgo de presentar mucosa ectópica que puede generar sangrado, ${ }^{11,12}$ infección, obstrucción intestinal ${ }^{13}$ y perforación. ${ }^{14}$ Visnjic y cols. reportaron un caso de estenosis de intestino delgado posterior a la reducción de una hernia estrangulada en un infante. ${ }^{15}$

\section{CONCLUSIÓN Y RECOMENDACIONES}

En el presente caso el paciente fue operado en el contexto de una cirugía ambulatoria en un hospital de segundo nivel por lo que se decidió no resecar el divertículo de Meckel incidental asintomático. En base a los hallazgos de la literatura médica nosotros recomendamos el manejo conservador en el lactante menor, comparando el riesgo de complicación de un Meckel incidental con la morbilidad postoperatoria de una resección intestinal en un lactante menor en una unidad hospitalaria de segundo nivel de atención.

\section{REFERENCIAS BIBLIOGRÁFICAS}

1. Pozo C, Enriquez M, Prats R, Domínguez C. Hernia de Littre. Reporte de un caso. Rev. Chilena de Cirugía. 2007; 59(4): p. 302-304.
2. Pampal A, Demirci E. Littre hernia in childhood: A case report with a brief review of the literature. African Journal of Paediatric Surgery. 2011; 8(2): p. 221-224.

3. Alarcón JR. Hernia de Littre. Colombia Médica. 2005; 36(4): p. 263-265.

4. Biel A, Vilallonga R, López I, Rodríguez N, Armengol M. Hernia de Littré: hallazgos inusitado en la reparación inguino-escrotral. Rev Esp Enferm Dg. 2010; 102(8): p. 506-513.

5. Skandalakis P, Zoras O, Skandalakis J, Mirilas P. Littré hernia: surgical anatomy, embryology, and technique of repair. Am Surg. 2006; 72(3): p. 238-243.

6. Citgez B, Yetkin G, Uludag M, Karakoc S, Akgun I, Ozsahin H. Littre's hernia an incarcerated ventral incisional hernia containing a strangulated meckel diverticulum: report of a case. Surg Today. 2011; 41(4): p. 576-578.

7. Ahmad K, Shaikh F, Ng S, Grace P. Laparoscopic port Littre's hernia: a rare complication of Meckel's diverticulum. Am J Surg. 2006; 191(1): p. 124-5.

8. Soares H, Felicio A, Berthier G. Hernia de Littré causando obstruçao intestinal. Rev Col Bras Cir. 2009; 36(2): p. 183-184.

9. Ramos M, Romero C, Rodríguez C, Rivas M, Girón J. Patología del Mesenterio. Isquemia Intestinal. Malformaciones intestinales. Lesiones vasculares del intestino delgado. Medicine. 2012; 11(4): p. 231-238.

10. Snyder C. Divertículo de Meckel. In Ashcraft, editor. Cirugía Pediatrica. México: McGrawHill Interamericana; 2000. p. 571.

11. Horkoff $M$, Chan N, Hunter J. A large incarcerated Meckel's diverticulum in an inguinal hernia. Int J Surg Case Rep. 2014; 5(12): p. 899-901.

12. Dunn T, Markgraf W. Littre hernia - incarcerated Meckel's diverticulum. Am J Surg. 1962;(103): p. 144-145.

13. Aláez-Chillarón A, Ramiro-Pérez C, RodríguezVelasco G, Calero-Amaro A, Latorre-Fragua R, Díez-Tabernilla $M$. Littré hernia: an unusual cause of intestinal obstrucción. Cir Cir. 2012; 80(2): p. 186-8.

14. Yagmur Y, Akbulut S, Ali M. Gastrointestinal perforation due to incarcerated Meckel's diverticulum in right femoral canal. World J Clin Cases. 2014; 2(6): p. 232-234.

15. Višnjić S, Car A, Kralj R. Stenosis of the small intestine after reduction of strangulated Littre hernia in an infant. Hernia. 2013; 17(2): p. 285-8. 